\title{
Sensor Selection for Collaborative Spectrum Sensing Using SVD-QR
}

\author{
Dejian $\mathrm{Li}^{1}$, Weixia Zou ${ }^{1}$, Zheng Zhou ${ }^{1}$ and Yabin $\mathrm{Ye}^{2}$ \\ 1. Wireless Network Lab, Beijing University of Posts and Telecommunications, Beijing, China \\ E-mail: lidejian09@gmail.com \\ 2. European Research Center, Huawei Technologies Duesseldorf GmbH
}

\begin{abstract}
Collaborative spectrum sensing enables opportunistic unlicensed access to the unused portions of the licensed spectrum. In this paper, we propose an effective sensor selection scheme for collaborative spectrum sensing over shadow fading channel using SVD-QR decomposition. Since shadow fading is correlated for closely spaced sensors, it is desired to select fewer independent sensors to reduce battery consumption and save bandwidth, while still adopting adequate sensors to make the sensing performance satisfied. The proposed scheme can reduce the redundancy in cognitive radio networks and does not rely on the position information of sensors. The simulation results illustrate that the SVD-QR algorithm outperforms correlation measure based selection method for spectrum sensing.
\end{abstract}

Index Terms-Sensor selection, SVD-QR, spectrum sensing, collaborative detection, cognitive radio

\section{INTRODUCTION}

In cognitive radio (CR), the energy detector [1] works well when the signal-to-noise ratio (SNR) is high. However, in wireless channels, signals often suffer from shadow fading, which may lead to a very low SNR. In this case, the energy detector might determine that a deeply shadowed channel is unoccupied, causing large interferences to the primary user.

In order to improve the reliability of spectrum sensing, collaborative spectrum sensing schemes has been proposed (e.g [2][3]). Collaborative spectrum sensing can alleviate the problem of corrupted detection by exploiting spatial diversity, and thus reduces the probability of interfering with primary users. It is effective to solve the hidden terminal problem.

In [4][5], it was found that the probability of error in collaborative spectrum sensing usually decreases as the number of collaborating users increases. However, there exists an error floor where the decrease of the probability of error is minor when the number of collaborating users increases further, for correlated samples. From a performance standpoint, one should use more secondary users in collaborative sensing to achieve as much performance gain as possible. From a complexity standpoint, one should use less secondary users in collaborative sensing, since the consumption of system resources, such as the total transmission power of the signal measurements, the

This work was supported by NSFC (60772021), National 863 Program (2009AA01Z262), Important National Science \& Technology Specific Projects (2009ZX03006-006/-009) and Korean Ministry of Knowledge Economy Project (IITA-2009-C1090-0902-0019). amount of overhead traffic and the size of memory required at the fusion center, grows approximately linearly with the number of collaborating users. Efficient collaborative schemes should be designed to reduce bandwidth requirements while maximizing the sensing reliability. Thus, it is desirable to use the minimum number of $\mathrm{CR}$ users (i.e. sensors) to achieve a satisfied sensing performance.

The SVD-QR algorithm was used in [6] to reduce information redundancy in Wireless Sensor Networks. It was found that there exists redundancy among the information collected from adjacent sensor nodes. We state that the energy sensing results from adjacent $\mathrm{CR}$ users in a cell for collaborative spectrum sensing might be very similar from each other, since the distance to the primary user and the shadow fading suffered are similar. There must be redundancy in the data reported by sensors in the neighborhood. Sensor selection for CR networks was studied in [7]. It introduced an approach to select sensors which are sufficiently spatially separated. However, sensors' positions or approximate positions should be known as priority for central node.

In this paper, we present a sensor selection scheme for collaborative energy sensing using SVD-QR decomposition without any position knowledge of sensors. Lognormal shadowing channel is examined. The objective is to select a set of sensors which experience uncorrelated shadow fading, and such a set of sensors can achieve higher performance in a subsequent sensing than a set of sensors selected only based on correlation measure in [7]. The SVD-QR algorithm can select uncorrelated sensors that perform reliable spectrum sensing using their local spectrum sensing results.

The rest of this paper is organized as follows. In Section II, the system model and spectrum sensing are briefly introduced. Collaborative Spectrum Sensing and performance metrics are also derived in section II. In section III, the sensor selection schemes based on SVD-QR and correlation measure are presented. Simulation results are presented and discussed in Section IV. Finally, we draw our conclusions in section V.

\section{Proposed Spectrum SENSING SCHEME}

\section{A. System Model}

We consider collaborative spectrum sensing in a centralized system. Assume that a CR system is composed of $K$ CR users. Each CR user individually performs the spectrum sensing and 
all CR users are strict synchronous. In the centralized systems, all the sensing results are reported to the central CR device that makes a decision on the presence of a primary user.

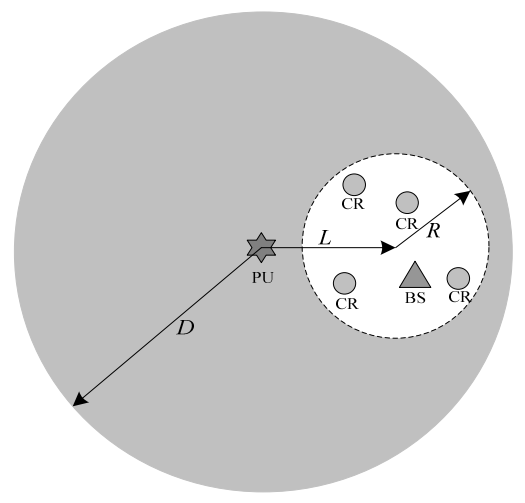

Fig. 1. Spectrum sensing structure of a cognitive radio network.

In Fig. 1, BS is the coordinating central CR device, which selects CRs to perform sensing and makes the final decision by fusing the local sensing results from the selected sensors. PU is a primary user. CR, also named sensor, is a secondary user when performs collaborative sensing. All CR users and a BS forming a circular cell are deployed randomly in a range presented in Fig. 1. We consider a single cell hence. $D$ is the primary user's radio range. The radius of the cell is $R$.

When the PU is transmitting, the main contributor to the decision static is the received signal energy from the PU, which depends on channel gain. We consider the channel gain as the combination of the path loss due to the distance and the shadow fading gain. Assume that the PU transmits with a fixed power Pt. With the simplified path loss model [3], the received signal energy from the PU during $T_{s}$ can be represented as

$$
E_{r}=\left(P_{t} \cdot T_{s}\right) \cdot \beta S^{-\gamma} \cdot \Psi
$$

where $\Psi$ is a shadow fading gain which follows lognormal distribution, $S$ is the distance between a CR user and the PU, $\gamma$ is the path loss exponent, and $\beta$ is the path loss unit constant.

\section{B. Energy Detection}

In this paper, we choose the energy detection for sensors, since it can simply be implemented and used even when the signal form of a primary user is unknown. The local spectrum sensing problem can be formulated as follows.

To detect a weak primary signal confined inside some a priori known bandwidth $\mathrm{B}$, one could pose a binary hypothesis testing problem as follows:

$$
\begin{aligned}
& \mathcal{H}_{0}: x(n)=v(n) \\
& \mathcal{H}_{1}: x(n)=s(n)+v(n), n=1,2, \ldots N,
\end{aligned}
$$

where $\mathcal{H}_{0}$ represents the absence of the primary signal, i.e., the received baseband complex signal $\mathrm{x}(\mathrm{n})$ contains only additive white Gaussian noise (AWGN), $v(n) \sim \mathcal{N}\left(0, \sigma_{v}^{2}\right) \cdot \mathcal{H}_{1}$ represents the presence of the primary signal, i.e., $x(n)$ consists of a primary signal $s(n)$ corrupted by $v(n)$. Moreover, $\mathrm{N}$ corresponds to the number of available measurements.
The noncoherent energy detector [2] is used in this paper. Let $\mathbf{x}=[x(1), x(2), \ldots, x(N)]^{T} \quad$ and $\quad \mathbf{s}=[s(1), s(2), \ldots, s(N)]^{T} \quad$. The decision rule in this case is given by

$$
T(\mathbf{x}) \triangleq \sum_{n=1}^{N}|x(n)|^{2} \underset{\substack{\mathcal{H}_{1} \\ \mathcal{H}_{0}}}{<} \gamma,
$$

Where $T(\mathbf{x})$ is the test statistic and $\gamma$ is the corresponding test threshold. Although $T(\mathbf{x})$ has a chi-square distribution, according to the central limit theorem, $T(\mathbf{x})$ is asymptotically normally distributed if $N$ is large enough ( $N \geq 20$ is often sufficient in practice). Specifically, for large $N$, we can model the statistics of $T(\mathbf{x})$ as follows:

$$
T(\mathbf{x}) \sim \begin{cases}N\left(N \sigma_{v}^{2}, 2 N \sigma_{v}^{4}\right) & \text { under } H_{0} \\ N\left(N \sigma_{v}^{2}+N p_{s}, 2 N \sigma_{v}^{4}+4 N \sigma_{v}^{2} p_{s}\right) & \text { under } H_{1}\end{cases}
$$

where $p_{s}=\|s\|^{2} / N$ represents the average primary signal power. In this way, for large $N$, the probability of false alarm, $P_{f}$, and the probability of detection, $P_{d}$, can be approximated as

$$
P_{f}=P\left(\mathcal{H}_{1} \mid \mathcal{H}_{0}\right)=Q\left(\frac{\gamma-N \sigma_{v}^{2}}{\sigma_{v}^{2} \sqrt{2 N}}\right)
$$

and

$$
P_{d}=P\left(\mathcal{H}_{1} \mid \mathcal{H}_{1}\right)=Q\left(\frac{\gamma-N \sigma_{v}^{2}-N p_{s}}{\sigma_{v} \sqrt{2 N \sigma_{v}^{2}+4 N p_{s}}}\right),
$$

respectively, where

$$
Q(x)=\frac{1}{\sqrt{2 \pi}} \int_{x}^{+\infty} e^{-\tau^{2} / 2} d \tau
$$

is the tail probability of a zero-mean unit-variance Gaussian random variable.

Given the probability of detection $P_{d}$, we get the missed detection probability $P_{m}$

$$
P_{m}=P\left(\mathcal{H}_{0} \mid \mathcal{H}_{1}\right)=1-P_{d}=1-Q\left(\frac{\gamma-N \sigma_{v}^{2}-N p_{s}}{\sigma_{v} \sqrt{2 N \sigma_{v}^{2}+4 N p_{s}}}\right)
$$

The signal-to-noise ratio is defined as

$$
S N R=\frac{p_{s}}{\sigma_{v}^{2}}=\frac{\|S\|^{2}}{N \sigma_{v}^{2}}
$$

\section{Collaborative Sensing}

In our approach, CR user's operating duration is divided into two parts in collaborative sensing: one is Sensor Selection Period and the other is Normal Period. In the Sensor Selection Period, all sensors perform local energy spectrum sensing and send the exact sensing results to the BS without making a decision. The BS selects the most significant sensors based on the received local observations using SVD-QR algorithm. In the Normal Period, the sensors that are selected to participate in collaborative spectrum sensing makes a local binary decision as the sensing result and only send one bit decision to BS. The Sensor Selection Period is much shorter than Normal Period when the mobility of the CR network is low, since the BS does not have to perform sensor selection frequently. 
For the decision fusion scheme, we choose OR rule. The BS infers the presence of the PU signal when there exists one CR user that has the local decision $\mathcal{H}_{1}$. It can be seen that the OR rule is very conservative for the CRs to access the licensed band [8]. As such, the chance of causing interference to the PU is minimized. We shall consider the OR rule in the sequel.

The false alarm probability of collaborative spectrum sensing based on the OR rule is given by [8]

$$
Q_{f}=1-\prod_{i=1}^{K}\left(1-P_{f}^{(i)}\right),
$$

where $P_{f}^{(i)}$ denotes the false alarm probability of the $i$ th CR in its local spectrum sensing. $K$ is the number of the sensors selected to perform collaborative sensing. The missed detection probability of collaborative spectrum sensing is given by

$$
Q_{m}=1-\prod_{i=1}^{K} P_{m}^{(i)},
$$

where $P_{m}^{(i)}$ denotes the missed detection probability of the ith $\mathrm{CR}$ in its local spectrum sensing.

Assume that every $\mathrm{CR}$ has the same noise level, i.e., for equation (4), all CR users has the identical $\sigma_{v}^{2}$. According to equation (4), it is seen that every $\mathrm{CR}$ achieves identical $P_{f}$ (i.e., $\left.P_{f}=P_{f}^{(i)}, \forall i=1,2, \ldots, K\right)$. The false alarm probability and the missed detection probability of cooperative spectrum sensing are then given by

$$
\begin{gathered}
Q_{f}=1-\left(1-P_{f}\right)^{K}, \\
Q_{m}=1-\prod_{i=1}^{K} P_{m}^{(i)},
\end{gathered}
$$

We stress that the condition for $(9) \sim(12)$ is that all selected CR users identify the availability of the licensed spectrum independently. To simplify the problem, CR users are considered as independent approximately if the correlations among them are small enough.

\section{Sensor SELECtion Algorithm}

\section{A. Sensor Selection Based on SVD-QR}

In order to perform reliable spectrum sensing, several sensors which experience (at least to some extent) uncorrelated fading, with respect to the possible signals they are sensing for, are required. The number of sensors to use is a trade-off between high reliability and high resources usage efficiency.

In the predefined CR network, the BS selects a few CR users as active sensing set before CR users send the binary decisions to the BS. Other CR users that are not selected by BS do not perform spectrum sensing during the Sensing Period.

The following SVD-QR algorithm used here is as same as the one designed for wireless sensor network in [6]. The algorithm is to select a set of independent data sets that minimize the residual error in a least-squares sense:

1) Given $P \in R^{m \times n}$, assume $n>m, \operatorname{rank}(P)=r \leq m$ denote the rank of $P$. Determine a numerical estimate $r^{\prime}$ of the rank of the data sets matrix $P$ by calculating the singular value decomposition

$$
P=U\left[\begin{array}{ll}
\Sigma & 0 \\
0 & 0
\end{array}\right] V^{T},
$$

where, $U$ is an $N \times N$ matrix of orthonormalized eigenvectors of $P P^{T}, V$ is an $m \times m$ matrix of orthonormalized eigenvectors of $P^{T} P$, and $\sum$ is the diagonal matrix $\Sigma=\operatorname{diag}\left(\sigma_{1}, \sigma_{2}, \ldots, \sigma_{r}\right)$, where $\sigma_{i}$ denotes the $i^{\text {th }}$ singular value of $P$, and $\sigma_{1} \geq \sigma_{2} \geq \cdots \geq \sigma_{r}>0$. Select $\hat{r} \leq r^{\prime}$.

2) Calculate a permutation matrix $\Pi$ such that the columns of the matrix $\Gamma_{1} \in R^{n \times \hat{r}}$ in

$$
P \Pi=\left[\Gamma_{1}, \Gamma_{2}\right]
$$

are independent. The permutation matrix $\Pi$ is obtained from the QR decomposition of the submatrix comprised of the right singular vectors, which correspond to the $\hat{r}$ ordered most-significant singular values.

In short, we select the data sets as the following:

- Decomposes $P$, from the SVD of $P$, save $V$.

- Observe $\Sigma$, Select an appropriate $\hat{r}$.

- Partition

$$
V=\left[\begin{array}{ll}
V_{11} & V_{12} \\
V_{21} & V_{22}
\end{array}\right]
$$

where $\quad V_{11} \in R^{\hat{r} \times \hat{r}} \quad, \quad V_{12} \in R^{\hat{r} \times(m-\hat{r})} \quad, \quad V_{21} \in R^{(m-\hat{r}) \times \hat{r}} \quad$,and $V_{22} \in R^{(m-\hat{r}) \times(m-\hat{r})}$. In many practical cases, $\sigma_{1}$ is much larger than $\sigma_{r^{\prime}}$; thus $\hat{r}$ can be chosen much smaller than the estimate $r^{\prime}$ of $\operatorname{rank}(\mathrm{P})$, even 1 .

- Using QR decomposition with column pivoting, determine $\Pi$ such that

$$
Q^{T}\left[V_{11}^{T}, V_{21}^{T}\right] \Pi=\left[R_{11}, R_{12}\right],
$$

where $Q$ is a unitary matrix, and $R_{11}$ and $R_{12}$ form an upper triangular matrix; and $\Pi$ is the permutation matrix, the column permutation $\Pi$ is chosen so that $\operatorname{abs}(\operatorname{diag}(R))$ is decreasing. In short, $\Pi$ corresponds to the $\hat{r}$ ordered most-significant sets.

Examples for how to use the SVD-QR decomposition for sensor selection are given in [6]. One of the advantages of SVD-QR is that it can solve two problems simultaneously: determine how many sensors are uncorrelated and identify which sensors are uncorrelated.

\section{B. Sensor Selection Based on Correlation Measure}

To give a comparison of spectrum sensing performance, we give a simple review of the existing sensor selection algorithm in [7]. The author of [7] presented three different algorithm to select a set of sensors that do not experience correlated shadow fading when perform spectrum sensing. The three algorithms for sensor selection use different amount of information of the sensors' positions. We choose one typical algorithm based on correlation measure for comparison. The algorithm is based on the following optimization problem:

$$
\begin{aligned}
\min & \sum_{i=1}^{M} \sum_{j=1}^{M} a_{i} a_{j} c_{i j} \\
\text { subject to } & \sum_{i=1}^{M} a_{i}=N \\
& a_{i} \in\{0,1\}, i=1, \ldots, M
\end{aligned}
$$

where $a_{i}$ represents activity of the sensor $i$ : $a_{i}=1$ if the sensor belongs to the active set and $a_{i}=0$ if the sensor belongs to the passive set. $M$ is the total number of sensors available for sensing and the user parameter $N$ is the desired number of 
sensors to use in the sensing. The term $c_{i j} \geq 0$ is a correlation measure between the sensors $i$ and $j$. The correlation measure could be a correlation function based on the Euclidian distance between the estimated positions of two sensors, such as $C(d)$

$$
C(d)=e^{-\alpha d}
$$

where $d$ is the distance and $\alpha$ is an environment parameter. In an urban non-line-of-sight environment, $\alpha=0.1204 / \mathrm{m}$, and in suburban environments $\alpha=0.002 / \mathrm{m}$. As stated in [7], A correlation of 0.2 is assumed "small enough", such that the decorrelation distance becomes $d_{0}=-\ln (0.2) / \alpha$. For an urban scenario, the decorrelation distance becomes $d_{0}=13 \mathrm{~m}$, that is, the minimum separation between sensors required for the shadowing correlation to fall below a determined threshold. More details about sensor selection based on correlation measure can be found in [7].

\section{Simulation Results}

This section contains simulation results of the sensor selection based on SVD-QR algorithm and correlation measure algorithm for collaborative spectrum sensing. The CR networks are formed as Fig. 1. We consider circular cell having a radius of $R=200 \mathrm{~m}$ (all positioning units are given in meters) with the PU placed in the center of the circle. In the CR cell, 100 sensors are distributed over the cell randomly uniformly. $L$ is set to $300 \mathrm{~m}$ and all sensors are assumed in the PU's transmission range. The distance between a sensor and the PU is calculated using their positions. The path loss and shadow fading parameters for equation (1) are set to $\gamma=3, \beta=1$, and the standard deviation of shadow fading $\Psi$ in logarithm is set to 5 $\mathrm{dB}$. The transmit power of PU is fixed at 1 . The PU is presented with probability 0.7 .

Assume that reporting channels that between sensors and the BS are errorless. Assume that the BS itself will not perform spectrum sensing. We suppose each sensor sends 100 spectrum sensing results to the BS in Sensor Selection Period. For equation (5) and (6), we set the number of available measurements $N=30$ and the average SNR is set to $-10 \mathrm{~dB}$.

It is seen in Fig. 2 that the 5 sensors selected from all 100 sensors using SVD-QR algorithm outperform the 5 sensors selected based on correlation measure in collaborative spectrum sensing. The performance is averaged over 500 sensor distribution realizations. In the SVD-QR sensor selection algorithm, we get a permutation matrix $\Pi$ from equation (16). Since the number of columns of $\Pi$ is same to the number of all the sensors, the ordering of the significance of the sensors in collaborative sensing can be obtained according to $\Pi$. We select 5 sensors according to the first 5 columns of $\Pi$.

From (5) to (6), for a given sensing time, the local spectrum sensing performance relies on SNR. However, according to (17) and (18), correlation measure based sensor selection algorithm only gets a set of sensors with largest mutual distances but ignores the SNR of the sensors. So the "distance penalty" for using lower correlation values would be large. This statement could be proved by the SNR of the sensors selected using SVD-QR and correlation measure, as seen in Fig. 3. Fig. 3 shows that the average SNR of the sensors selected by
SVD-QR is much higher than that of sensors selected by correlation measure. This explains why the sensors selected by SVD-QR achieve a much higher performance in spectrum sensing.

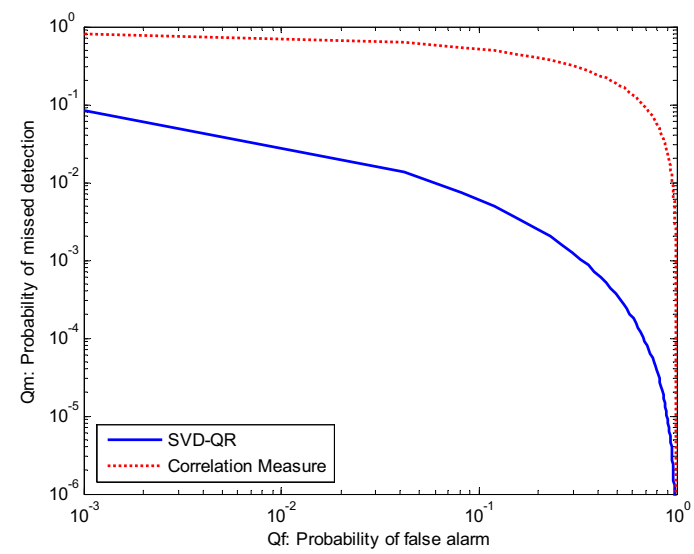

Fig. 2. Collaborative spectrum sensing performance comparison of 5 sensors selected based on SVD-QR and correlation measure.

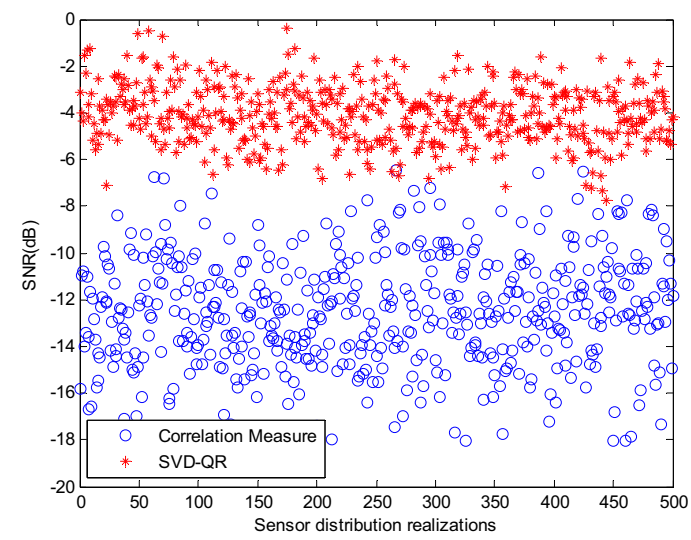

Fig. 3. The average SNR of the 5 sensors selected based on SVD-QR and correlation measure for 500 sensor distribution realizations.

Fig. 4 shows the minimum distance of the distances among the 5 sensors selected based on SVD-QR and correlation measure for 500 sensor distributions. Decorrelation distance $d_{0}=13 \mathrm{~m}$ is also plotted in Fig. 4 as the horizontal line. An interesting phenomenon in Fig. 4 is that the 5 sensors selected from 100 densely distributed sensors by SVD-QR are separated by considerable distance, though there is none position information of sensors in SVD-QR. There are only 25 realizations in which the minimum distance among the sensors selected by SVD-QR fall below the decorrelation distance. That means, for 500 sensor distribution realizations, the correlations calculated by (18) of the 5 sensors selected by SVD-QR are all smaller than 0.2 in $95 \%$ realizations. In other word, using the correlation model depicted by (18), the SVD-QR algorithm can select a few uncorrelated sensor without the information of their positions.

Fig. 5 shows the minimum distance of the distances among the 10 sensors selected based on SVD-QR and correlation measure without any change to the system parameters given 
above. As seen in Fig. 5, if the target number of sensor selection fixed to 10, the minimum distance of the sensors selected based on two algorithms both decrease significantly. The number of the minimum distance among the sensors selected by SVD-QR falls below the decorrelation distance increased up to 94 . Under such conditions, we can not use (11) (12) to get the theoretical performance of collaborative sensing, since the sensors selected by SVD-QR are not independent according to the correlation model depicted by (18). This tells us that we can not fix the target number of sensors to be selected for different sensor distributions. If we fix the target number, the two problems of how many sensors are uncorrelated and which sensors are uncorrelated will be separated. The SVD-QR algorithm can solve the two problems simultaneously.

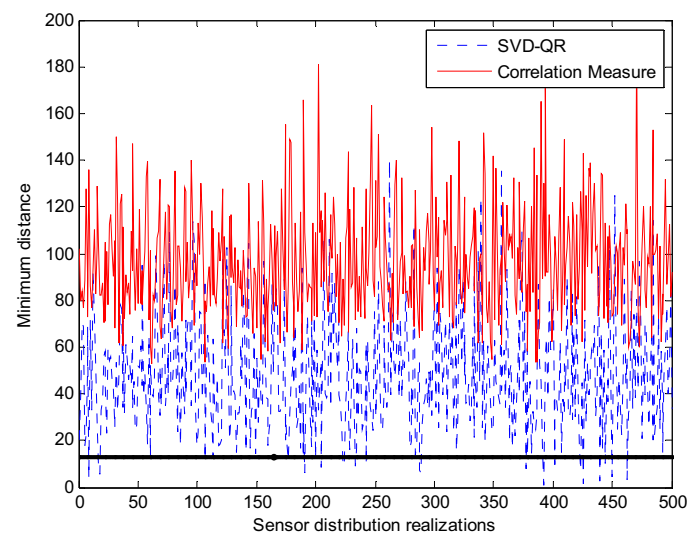

Fig. 4. Minimum distance of the distances among the $\mathbf{5}$ sensors selected based on SVD-QR and correlation measure for 500 sensor distributions.

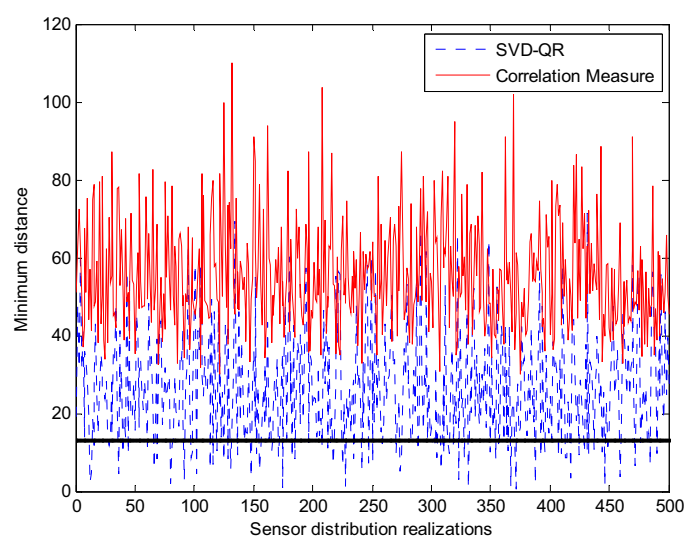

Fig. 5. Minimum distance of the distances among the $\mathbf{1 0}$ sensors selected based on SVD-QR and correlation measure for 500 sensor distributions.

\section{CONCLUSION}

In this paper, we have proposed an effective sensor selection scheme for collaborative spectrum sensing based on SVD-QR decomposition. There exists redundancy among CR users that suffer shadow fading and the most significant sensors that suffers uncorrelated shadow fading should be selected to achieve a satisfied sensing performance. As a result, the SVD-QR sensor selection algorithm outperforms the correlation measure based sensor selection algorithm. Future work includes theoretical analysis on the relationship of the SVD-QR and the correlation of sensors.

\section{REFERENCES}

[1] H. Urkowitz, "Energy detection of unknown deterministic signals," Proc.IEEE, vol. 55, pp. 523-531, Apr. 1967.

[2] Zhi Quan; Shuguang Cui; Poor, H.; Sayed, A.; "Collaborative Wideband sensing for Cognitive radios, "Signal Processing Magazine, IEEE Volume 25, Issue 6, Page(s):60 - 73, November 2008

[3] Sang Soo Jeong; Wha Sook Jeon; Dong Geun Jeong; "Collaborative Spectrum Sensing for Multiuser Cignitive Radio Systems," Vehicular Technology, IEEE Transactions on Volume 58, Issue 5, Page(s):2564 2569 , Jun 2009

[4] E. Visotsky, S. Kuffner, and R. Peterson, "On collaborative detectionof TV transmissions in support of dynamic spectrum sharing," in Proc.IEEE 1 st Symposium on Dynamic Spectrum Access Networks, 2005.

[5] A. Ghasemi and E. S. Sousa, "Asymptotic performance of collaborative spectrum sensing under correlated log-normal shadowing," IEEE Commun. Lett., vol. 11, pp. 34-36, Jan. 2007.

[6] Qilian Liang; Lingming Wang; "Redundancy Reduction in Wireless Sensor Networks using SVD-QR,'Military Communications Conference, 2005. MILCOM 2005. IEEE 17-20, Page(s):1857- 1861 Vol. 3, Oct. 2005

[7] Yngve Selen; Tullberg, H.; Kronander, J.; "Sensor Selection for Cooperative Spectrum Sensing, "New Frontiers in Dynamic Spectrum Access Networks, 2008. DySPAN 2008. 3rd IEEE Symposium on 14-17 Page(s): 1 - 11, Oct. 2008

[8] Wei Zhang; Ben Letaief, K; "Cooperative Communications for Cognitive Radio Networks," Proceedings of the IEEE Volume 97, Issue 5, Page(s):878 - 893, May 2009 